\title{
Eisenmenger Syndrome
}

National Cancer Institute

\section{Source}

National Cancer Institute. Eisenmenger Syndrome. NCI Thesaurus. Code C84390.

A syndrome characterized by the reversal of a septal defect-associated left-to-right shunt due to the resulting secondary pulmonary hypertension. The presence of the pulmonary hypertension causes increased pressure in the right heart chambers resulting in the reversal of the shunt into a right-to-left shunt. Signs and symptoms include cyanosis, pulmonary hypertension, heart failure, and high red blood cell count. 